\title{
A MU MU COLLIDER CAPTURE SOLENOID SYSTEM FOR PIONS FROM A TILTED TARGET*
}

\author{
M. A. Green ${ }^{\mathrm{a}}$, and R. B Palmer ${ }^{\mathrm{b}}$ \\ a. Lawrence Berkeley National Laboratory, Berkeley CA 94720, USA \\ b. Brookhaven National Laboratory, Upton NY 11973, USA
}

\begin{abstract}
In order to generate muon beams for a muon Collider, pions produced in a target must be captured before they can go through phase rotation and decay into muons. A $20 \mathrm{~T}$ solenoid with a clear bore of $150 \mathrm{~mm}$ can capture particles with a transverse momentum of $225 \mathrm{MeV} / \mathrm{c}$ or less. As captured particles leave the capture solenoid, the induction is decreased to $5 \mathrm{~T}$ while the beam pipe diameter increases to $300 \mathrm{~mm}$. Phase rotation and decay of the pions will occur at $5 \mathrm{~T}$. The capture solenoid and the solenoids just downstream from the capture solenoid are subject to high heat loads (up to $2.4 \mathrm{MW}$ ). The conventional inner solenoid of the $20 \mathrm{~T}$ hybrid magnet system and the water cooled absorber will absorb most of the beam power from the protons thus protecting the superconducting solenoid system from heating. Tilting the target and the incoming beam about 150 milliradians with respect to the solenoid axis appears to increase the net pion capture by about sixty percent. This paper describes approaches for capturing muons from a tilted target while getting rid of the spent proton beam.
\end{abstract}

\section{BACKGROUND}

Capture of the pions from the target can occur in a solenoid of any induction provided the solenoid bore is large enough[1,2]. A capture induction of $20 \mathrm{~T}$ within a radius of $75 \mathrm{~mm}$ is needed to capture pions with a transverse momentum up to $225 \mathrm{MeV} / \mathrm{c}$. Once the pions have been captured in the solenoidal field, the channel solenoidal induction is reduced to $5 \mathrm{~T}$. Because some of the particle transverse momentum is transferred to forward momentum, the clear bore radius of the $5 \mathrm{~T}$ solenoid is only $150 \mathrm{~mm}$. The pions are transferred to a system of phase rotation rf cavities that speed up the low momentum particles and slow down the high momentum particles so that the particles travel bunched in time to the muon cooling system. Ninety-nine percent of the pions decay to muons within the first 80 meters.

The hybrid capture magnet option was selected for the following reasons: 1) When a superconducting outsert solenoid is used the operating power for the water cooled solenoid is lower. 2) The current density in the water cooled solenoid can be low enough to insure that its life time will be long (at least $25000 \mathrm{hr}$ 3) Additional space inside the water cooled solenoid can be made available for a heavy metal water cooled shield, which reduces the incident energy from the target into the water cooled solenoid and the surrounding superconducting solenoid.

\footnotetext{
* This work was performed with the support of the Office of High Energy and Nuclear Physics, United States Department of Energy under contract numbers DE-AC03-76SF00098 and DE-AC02$76 \mathrm{CH} 00016$
}

\section{THE TILTED TARGET}

Calculations of pion yield from a target suggest that tilting the incident proton beam and target with respect to the solenoid axis increases the pion yield for a given input proton intensity to the target. Pions produced in the upstream end of the target are not recaptured by the target downstream from the point where they were created. Simulations of pion production suggest that the pion production is maximized at a tilt angle of about 150 milliradians (see Figure 1). Above the 150 milliradian tilt angle the transverse momentum of some of the pions in the target becomes too high for capture to occur.

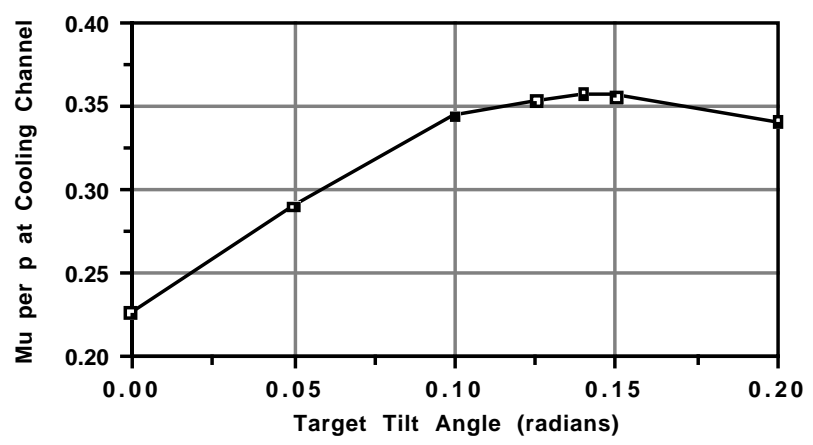

Figure. 1. Muon Production at the Cooling Channel Entrance for Optimized Targets as a Function of Target Tilt Angle

An increase in the yield of pions from the target means that the input beam power from the driver can be reduced, for instance, from 3.8 MW to about 2.4 MW. Even with improved efficiency, a copper target will absorb about $230 \mathrm{~kW}$ of energy from the proton beam. Cooling the target is a difficult proposition, which will not be addressed here. Tilting the target and the input proton beam allows virtually all of the beam energy that has not been transferred to the captured pions to be absorbed in the capture solenoid. The superconducting magnet will absorb less than $500 \mathrm{~W}$ of the energy from the beam.

\section{BITTER SOLENOID INSERT}

In this study, a Bitter solenoid insert that has an inner radius of $120 \mathrm{~mm}$ has been assumed. The extra inner bore radius allows a water cooled heavy metal (tungsten or some other high $\mathrm{Z}$, high density metal) shield that is 30 $\mathrm{mm}$ thick to be inserted around the target region. An extra $15 \mathrm{~mm}$ on the inside of the solenoid is allotted for water cooling channels for the shield. The Bitter solenoid insert outer radius has been set at $345 \mathrm{~mm}$. The superconducting outsert solenoid cryostat starts at a radius 
of $370 \mathrm{~mm}$. The $25 \mathrm{~mm}$ between the Bitter solenoid and the cryostat wall is allocated for water cooling. Much of the space at the ends of the Bitter insert solenoid should be filled with a water cooled shield to protect the superconducting solenoids on the outside from particles coming from the target.

The Bitter solenoid insert is divided along its length into three regions, each with a different current density[4]. The gradation of the current density in the windings allows for the magnetic field to be shaped along the solenoid axis, in the high field region. The current density in the Bitter solenoid section can be changed by changing the thickness of the plates in the solenoid. Thick palates have a lower current density than thin plates.

A Bitter solenoid is characterized by a current density in the winding that varies inversely as radius. The highest current density is found on the inside radius of the solenoid The highest current density part of the highest current density coil has a current density of 108.8 A per square $\mathrm{mm}$, which is lower than the highest current density sections of some of the high field hybrid solenoids[5]. Most of the magnet power is dissipated in the highest current density section. The cooling system must handle about 15.6 MW (13.3 MW of resistive heating plus $2.3 \mathrm{MW}$ of beam power).

\section{SUPERCONDUCTING OUTSERT SOLENOID}

The water cooled Bitter insert solenoid is surrounded by a superconducting outsert solenoid system. The combination of the Bitter magnet insert and the superconducting outsert generated a magnetic induction of $20 \mathrm{~T}$ in the region of the target. The superconducting outsert is defined as the three coils that are immediately outside of the Bitter magnet insert. Other superconducting solenoids downstream from the outsert coils form the transition region that shapes the magnetic field between the target and the phase rotation system. Within the transition region solenoids, the spent beam from the target must be dealt with. The energy absorber must be thick enough to prevent heating of the superconductor in the outsert and transition region coils. As a result, the energy absorber will extend some distance downstream from the target. The material that absorbs particles from the target will become radioactive. The superconducting magnet around the target, the Bitter solenoid and energy absorption material should be split so that it can be taken apart so that the radioactive material inside magnet can be dealt with. The split in the magnet could occur at the end of the transition solenoids.

The outsert solenoid cryostat starts at a radius of 370 $\mathrm{mm}$. The outsert coil alone is will produce a central induction of about 7.5 T. Operating under this condition, the outsert solenoid can be made from niobium titanium in a copper matrix operating at $2.0 \mathrm{~K}$. The outsert solenoid has three coils with an inner radius of $400 \mathrm{~mm}$. The outer radius of these coils is about $540 \mathrm{~mm}$. The peak magnetic induction in the outsert coil is about $8.3 \mathrm{~T}$.

\section{TRANSFER SOLENOID SYSTEM}

The transfer solenoid system guides the magnetic field so that the magnetic induction decreases in a smooth fashion from $20 \mathrm{~T}$ to $5 \mathrm{~T}$ at the start of the phase rotation channel. A transfer of the captured pions from the $20 \mathrm{~T}$ target region to the $5 \mathrm{~T}$ beam transport and phase rotation region requires an increase in the minimum beam pipe radius to $150 \mathrm{~mm}$.

Once the solenoid magnetic induction has been brought down to the field in the pion decay channel or phase rotation channel, the diameter of the solenoid coils must be changed to match the diameter of the coils in the decay channel solenoids or the solenoids in the phase rotation cavities. The change in solenoid diameter must be accomplished without altering the desired induction along the solenoid axis.

The option shown in Figure 2 has the final $5 \mathrm{~T}$ solenoid coil inside diameter set at $375 \mathrm{~mm}$. The system is suitable for transfer into phase rotation cavities that have their 5T solenoids inside the cavities. Solenoids inside the cavities is suitable if the acceleration gaps in the cavity are kept relatively small (below $110 \mathrm{~mm}$ ). This option shows the spent proton being captured within the superconducting transition magnet section.

A plot of magnetic induction along the axis of the capture and transfer solenoids as a function of distance from the end of the target is shown in Figure 3. Figure 3 shows that there is a smooth transition of the magnetic induction from the end of the target to the start of the first phase rotation cavity (with a $5 \mathrm{~T}$ solenoid within it).

Table 1 shows some parameters for the capture and transfer solenoid options. About 15.5 MJ of the system stored magnetic energy is due to the current that flows in the water cooled Bitter insert coils. The Bitter solenoid is inductively coupled with the superconducting solenoids. A shut down of the Bitter solenoid will increase the current in the superconducting magnets. This effect should be tolerable provided the superconducting solenoids are well cooled. A loss of current in the Bitter solenoids system will probably cause the superconducting solenoids to turn normal.

Table I: Capture Solenoid Parameters

$\begin{array}{lr}\text { Magnet Length to } \mathrm{x}=3 \mathrm{~m}(\mathrm{~m}) & 3.92 \\ \text { Cryostat OD at } \mathrm{x}=0(\mathrm{~m}) & 1.50 \\ \text { Cryostat OD at } \mathrm{x}=3 \mathrm{~m}(\mathrm{~m}) & 0.70 \\ \text { Bitter Magnet OD }(\mathrm{m}) & 0.69 \\ \text { Induction at Target }(\mathrm{T}) & 20.32 \\ \text { Induction at Target from Bitter Magnet (T) } & 12.86 \\ \text { Induction at Target from S/C Magnet (T) } & 7.46 \\ \text { Target Region Length (m) } & 0.23 \\ \text { Bitter Magnet Peak Current Density (A mm) } & 108.8 \\ \text { Bitter Magnet Power }(\mathrm{MW}) & \sim 13.0 \\ \text { Beam Tube ID at } \mathrm{x}=0(\mathrm{~m}) & 0.15 \\ \text { Beam Tube ID at } \mathrm{x}=3 \mathrm{~m}(\mathrm{~m}) & 0.30 \\ \text { Transition Length }(\mathrm{m}) & 1.10 \\ \text { Transport Channel Induction }(\mathrm{T}) & 5.0 \\ \text { Stored Energy to } \mathrm{x}=3 \text { meters }(\mathrm{MJ}) & 59.2 \\ \text { Stored Energy S/C Magnet to x }=3 \mathrm{~m}(\mathrm{MJ}) & 43.7\end{array}$




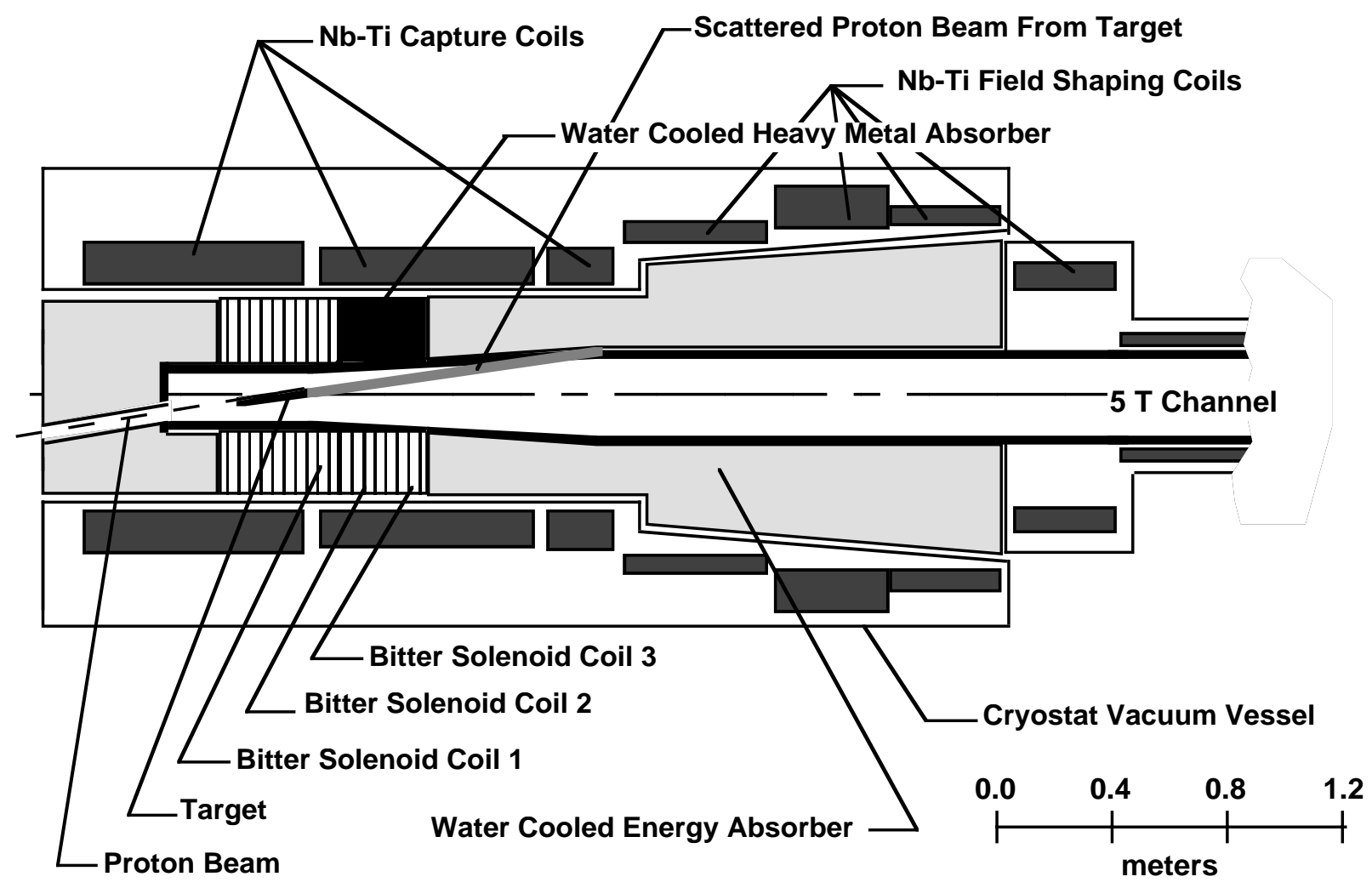

Figure 2 A 20 T Hybrid Capture Solenoid with a Transfer Solenoid System to $5 \mathrm{~T}$ in Phase Rotation RF Cavities

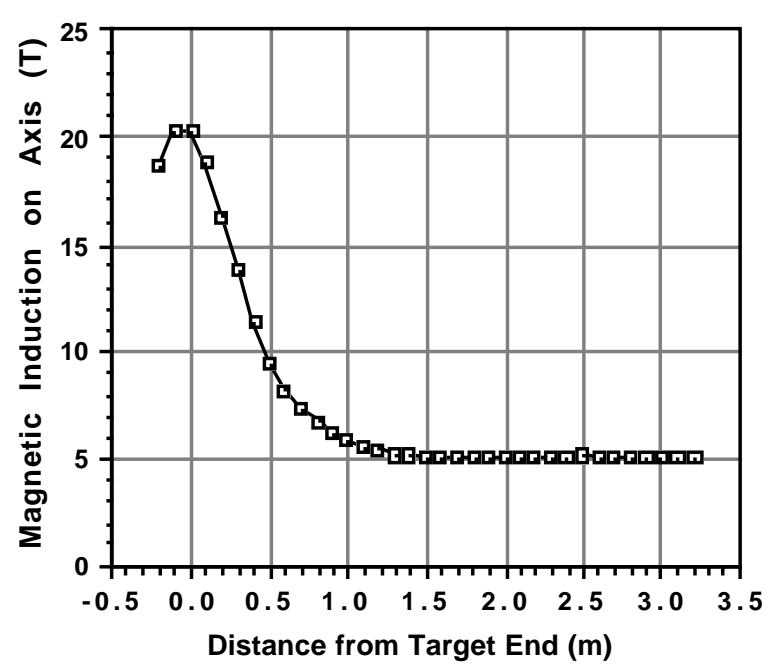

Figure 3 The On Axis Magnetic Induction as a Function of Distance from the Target in the Capture Solenoid System

\section{CONCLUSIONS}

A hybrid solenoid that uses a Bitter water cooled solenoid combined with a superconducting outsert solenoid appears to be an attractive magnet system for capturing and transporting pions generated in a target struck by a high energy proton beam. The hybrid solenoid permits one to shape the magnetic field in a way that is desirable for pion capture and transport to a phase rotation system. Tilting the target will increase the number of pion captured by about sixty percent. The problem of high heat loads from uncaptured particles can be solved with enough water cooled absorber inside of the superconducting magnet system. The water cooled insert solenoid is part of the energy absorption system. An advantage of the tilted target system is that the spent proton beam and any high energy electrons produced at the target are disposed of before they reach the phase rotation system.

\section{REFERENCES}

[1] N. V. Mokhov, R. J. Noble and A. Van Ginneken, "Targetry and Collection Optimization for Muon Colliders," AIP Conference Proceedings 372 (1996)

[2] M. A. Green, "Some Options for the Muon Collider Capture and Decay Solenoids," AIP Conference Proceedings 372 (1996)

[3] " $\mu^{+} \mu$ Collider, A Feasibility Study," by the $\mu^{+} \mu$ Collider Collaboration, BNL-52503, July 1996

[4] R. J. Weggel, Collector Magnets for $\mathrm{Mu}^{+}-\mathrm{Mu}^{-}$ Colliders, an unpublished report concerning preliminary design studies of capture magnets system for $2 \mathrm{TeV}$ on $2 \mathrm{TeV}$ and a demonstration collider

[5] J. R. Miller, M. D. Bird, S. Bole, et al, "An Overview of the 45T Hybrid Magnet System for the National High Field Magnet Laboratory," IEEE Trans. Mag 30 , No. 4, p 1563, (1994) 